\title{
Agricultural intensification and biodiversity partitioning in European landscapes comparing plants, carabids, and birds
}

\author{
Andreas Flohre, ${ }^{1,10}$ Christina Fischer, ${ }^{1}$ Tsipe Aavik, ${ }^{2}$ Jan Bengtsson, ${ }^{3}$ Frank Berendse, ${ }^{4}$ Riccardo Bommarco, ${ }^{3}$ \\ Piotr Ceryngier, ${ }^{5}$ Lars W. Clement, ${ }^{6}$ Christopher Dennis, ${ }^{7}$ Sönke Eggers, ${ }^{3}$ Mark Emmerson, ${ }^{7}$ Flavia Geiger, ${ }^{4}$ \\ Irene Guerrero, ${ }^{8}$ Violetta Hawro, ${ }^{5}$ Pablo Inchausti, ${ }^{9} \mathrm{JaAn}_{\text {Litra}},{ }^{2}$ Manuel B. Morales, ${ }^{8}$ Juan J. Oñate, 8 \\ Tomas Pärt, ${ }^{3}$ Wolfgang W. Weisser,${ }^{6}$ Camilla Winquist, ${ }^{3}$ Carsten Thies, ${ }^{1}$ and Teja Tscharntke ${ }^{1}$ \\ ${ }^{1}$ Agroecology, Department of Crop Science, Georg-August-University, Waldweg 26, 37073 Göttingen, Germany \\ ${ }^{2}$ Institute of Ecology and Earth Sciences, University of Tartu, Lai 40, Tartu EE51005, Estonia \\ ${ }^{3}$ Department of Ecology, Swedish University of Agricultural Sciences, P.O. Box 7044, 75007 Uppsala, Sweden \\ ${ }^{4}$ Nature Conservation and Plant Ecology Group, P.O. Box 47, Wageningen University, 6700 AA Wageningen, The Netherlands \\ ${ }^{5}$ Centre for Ecological Research, Polish Academy of Sciences, CBE PAN, Dziekanow Lesny, Konopnickiej 1, \\ 05-092 Lomianki, Poland \\ ${ }^{6}$ Institute of Ecology, Friedrich-Schiller-University Jena, Dornburger Straße 159, 07743 Jena, Germany \\ ${ }^{7}$ Department of Zoology, Ecology and Plant Sciences, University College Cork, Distillery Fields, Cork, Ireland \\ ${ }^{8}$ Department of Ecology, c/Darwin, 2, Universidad Autónoma de Madrid, Ciudad Universitaria de Cantoblanco, \\ 28049 Madrid, Spain \\ ${ }^{9}$ Centre for Biological Studies of Chizé CNRS, 79360 Villiers-en-Bois, France
}

\begin{abstract}
Effects of agricultural intensification (AI) on biodiversity are often assessed on the plot scale, although processes determining diversity also operate on larger spatial scales. Here, we analyzed the diversity of vascular plants, carabid beetles, and birds in agricultural landscapes in cereal crop fields at the field $(n=1350)$, farm $(n=270)$, and European-region $(n=$ 9) scale. We partitioned diversity into its additive components $\alpha, \beta$, and $\gamma$, and assessed the relative contribution of $\beta$ diversity to total species richness at each spatial scale. AI was determined using pesticide and fertilizer inputs, as well as tillage operations and categorized into low, medium, and high levels. As AI was not significantly related to landscape complexity, we could disentangle potential AI effects on local vs. landscape community homogenization. AI negatively affected the species richness of plants and birds, but not carabid beetles, at all spatial scales. Hence, local AI was closely correlated to $\beta$ diversity on larger scales up to the farm and region level, and thereby was an indicator of farm- and region-wide biodiversity losses. At the scale of farms (12.83-20.52\%) and regions (68.34-80.18\%), $\beta$ diversity accounted for the major part of the total species richness for all three taxa, indicating great dissimilarity in environmental conditions on larger spatial scales. For plants, relative importance of $\alpha$ diversity decreased with AI, while relative importance of $\beta$ diversity on the farm scale increased with AI for carabids and birds. Hence, and in contrast to our expectations, AI does not necessarily homogenize local communities, presumably due to the heterogeneity of farming practices. In conclusion, a more detailed understanding of AI effects on diversity patterns of various taxa and at multiple spatial scales would contribute to more efficient agrienvironmental schemes in agroecosystems.
\end{abstract}

Key words: agricultural intensification; alpha diversity; beta diversity; biodiversity patterns; cereal crop fields; community homogenization; landscape ecology; Western Europe.

\section{INTRODUCTION}

Global croplands, plantations, and pastures have expanded significantly in the last decades (Foley et al. 2005). This has led to dramatic changes in the spatial structure of agricultural landscapes in Western Europe. Formerly, structurally diverse landscapes have often been converted to simple landscapes mainly consisting of intensively managed agricultural units (Robinson and Sutherland 2002, Benton et al. 2003). Such agricultural

Manuscript received 29 March 2010; revised 15 October 2010; accepted 22 October 2010. Corresponding Editor: V. C. Radeloff.

${ }^{10}$ E-mail: aflohre@gwdg.de intensification (AI) is featured by higher pesticide and fertilizer inputs, tillage operations, and livestock densities, as well as an increasing simplification of agricultural landscapes, and was accompanied by a profound loss of biodiversity (Matson et al. 1997, Stoate et al. 2001, Bengtsson et al. 2005, Foley et al. 2005, Tscharntke et al. 2005, Billeter et al. 2008). While effects of agricultural intensification have been a major topic in ecological research in the last decade, they have mainly been studied at the plot spatial scale (spatial scales are henceforth addressed simply as scale), although processes determining diversity operate at different spatial scales (Crawley and Harral 2001, Collins et al. 2002, Weiher and Howe 2003, Aavik and Liira 2010), and 


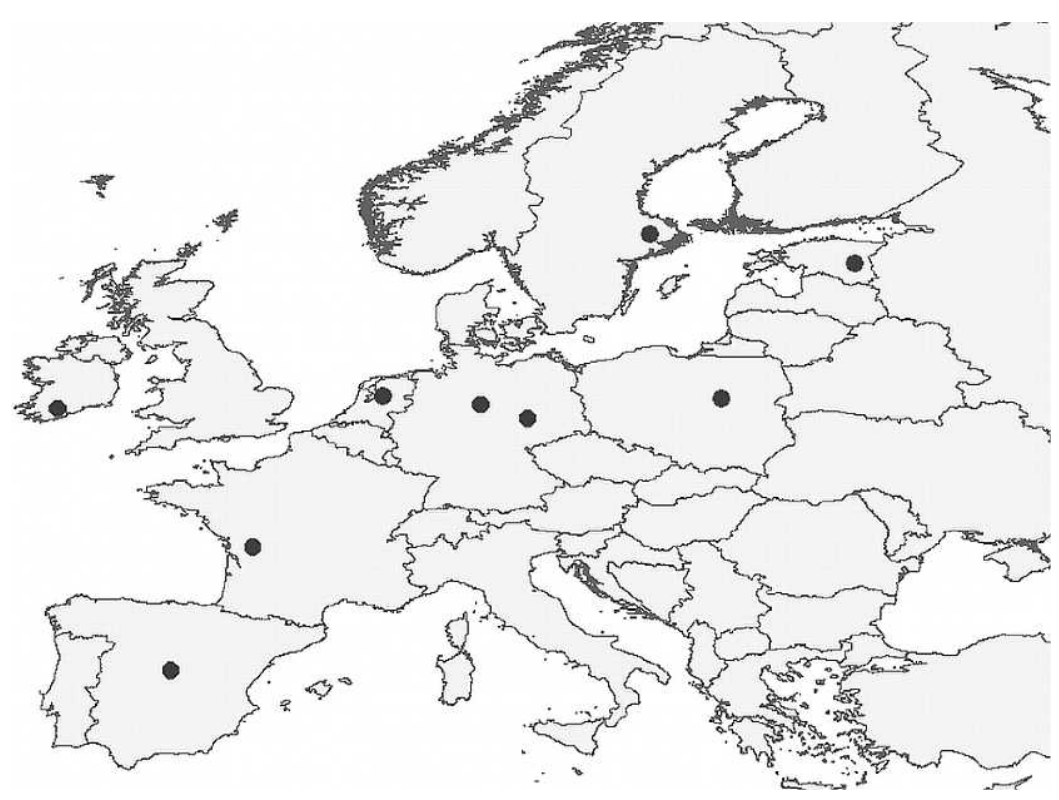

FIG. 1. Locations of the study regions, indicated by black dots in Sweden, Estonia, Ireland, The Netherlands, Germany (Göttingen), Germany (Jena), Poland, France, and Spain (from Geiger et al. 2010).

assessing diversity merely at one scale can be misleading (Tylianakis et al. 2006). For example, AI could lead to a homogenization of communities on, e.g., the field scale, which could have profound effects on the species turnover (i.e., the dissimilarity in species composition between plots) between fields and hence to species richness on larger scales (Cadotte 2006). However, one has to keep in mind that $\mathrm{AI}$ also acts on different spatial scales, e.g., intensification on a landscape level vs. intensification on the field (Tscharntke et al. 2005). Therefore, when addressing agricultural intensification at the field scale, one has to also acknowledge the landscape context.

A useful tool to evaluate diversity at different spatial scales is the additive partitioning approach (Lande 1996, Veech et al. 2002, Crist et al. 2003, Legendre et al. 2005, Clough et al. 2007): The total diversity of a sampling unit $(\gamma)$ can be separated into different components: $\alpha$ diversity, which is the average diversity on a plot scale, and $\beta$ diversity, which is the between-plot diversity. Thus, $\beta$ diversity is a measure of variation in species composition between plots. Beta diversity between plots and regions has been shown to account for up to $80 \%$ of the total species richness of arthropods (Tylianakis et al. 2006, Clough et al. 2007) or plants (Wagner et al. 2000, Gabriel et al. 2006). A more thorough understanding of the spatial organization of farmland diversity could give important insights into associated ecosystem processes and human-related drivers of species loss at different scales.

From an applied point of view, a more thorough understanding may be useful for selecting the appropriate scale for conservation efforts (e.g., agri-environmental schemes; Gering et al. 2003, Chandy et al. 2006) or for the identification of flexible conservation strategies (Gabriel et al. 2006, Diekötter et al. 2008) for different taxa.

Here, we applied additive partitioning of diversity to a data set from a biodiversity survey conducted in cereal crop fields across nine regions in Europe. We analyzed the effects of AI and landscape context on species richness of plants, carabid beetles, and breeding birds in agricultural landscapes and hypothesized: (1) AI has a negative effect on species richness of all taxa, with $\beta$ diversity making the major contribution to total diversity; (2) the three species groups respond differentially to AI on different spatial scales; (3) AI homogenizes communities across fields, and this homogenization negatively affects species turnover between fields and regions; and (4) local AI is not only related to local biodiversity losses, but also to losses at larger spatial scales.

\section{Methods}

\section{Study area and design}

The study was conducted in nine European regions (Estonia, France, East and West Germany separately, Ireland, Netherlands, Poland, Spain, and Sweden; Fig. 1) covering a north-south gradient from Sweden and Estonia to Spain, and a west-east gradient from Ireland to Poland in the context of the collaborative AGRIPOPES project (further details available online). ${ }^{11}$ In each region we selected 30 farms differing in the level of agricultural intensification (AI). Farms were situated in a $50 \times 50 \mathrm{~km}$ region to standardize spatial scales and account for larger edaphic or topographic gradients.

\footnotetext{
11 http://www.agripopes.net $\rangle$
} 
Each farm comprised (ideally) five cereal crop fields (see Geiger et al. 2010) for sampling of vascular plants and carabids and a $500 \times 500 \mathrm{~m}$ square for sampling of breeding birds centered on the largest field of each farm. When one farm could not provide five fields, five sampling points with maximum distance to each other were chosen. Each sampling point consisted of three vegetation plots in a row, with a distance of $5 \mathrm{~m}$ between each one, and one pitfall trap (Schmidt et al. 2006) placed in the middle of each of the outer vegetation plots. Plots were situated parallel to the field boundary with $10 \mathrm{~m}$ distance between field margin and sampling point.

\section{Sampling of organisms}

At the flowering stage of winter wheat (mid June) the number of vascular-plant species was counted in the vegetation plots (each $2 \times 2 \mathrm{~m}^{2}$, three plots per field). The number of carabid species was counted using two pitfall traps per plot (diameter $9.5 \mathrm{~cm}$, depth $15.4 \mathrm{~cm}$ ), opened for two one-week periods (one week after spike appearance and at the milk-ripening stage of winter wheat, respectively). Roofs made of cardboard fixed with needles prevented flooding by rain. The trapping fluid was $50 \%$ ethylene glycol. Carabids were stored in $70 \%$ ethanol and identified up to species level. We identified and counted all the species caught in one trap randomly selected from each pair of traps.

We used a simplified version of the British Trust for Ornithology's (BTO) Common Bird Census (Bibby et al. 1992) for bird species recording. All bird species were mapped in a $500 \times 500 \mathrm{~m}^{2}$ survey plot on each farm centered on one focal field. Mapping included three early morning visits between April and June. Windy, cloudy, or rainy weather was avoided. Birds that merely flew over the area without showing any behavior that indicated breeding or foraging/hunting activity were excluded. Breeding-bird territories were determined using the three survey rounds (for details see Appendix A). In some cases, not only the focal field, but also parts of the surrounding landscape were sampled. Our bird surveys thus were not restricted to arable bird species and include species from seminatural habitats next to the focal fields within the $500 \times 500 \mathrm{~m}$ square, to ensure that possible indirect effects of AI on the landscape structure are included in the analysis.

We are aware that the low sampling effort potentially underestimates local diversity and thus might overestimate $\beta$ diversities. However, we were mainly interested in the correlations between $\alpha, \beta$, and $\gamma$ diversity and agricultural intensification. Here, a standardized sampling protocol is most important and a potential underestimation of local diversity would enter the statistical analysis merely as a systematical error.

\section{Agricultural intensification index}

Agricultural intensification (AI) was measured using a standardized questionnaire on pesticide and fertilizer (mineral and organic) applications, tillage operations, and mechanical weed control, filled in by the farmers after harvest.

All of these indicators are known to have the potential to severely influence communities. The effects of fertilization on species richness and community composition of plants are well studied (Pysek and Leps 1991, Gough et al. 2000, Reich 2009). Likewise, the effects of pesticides on plant, carabid, and bird communities are well studied (Newton 2004, Geiger et al. 2010). Plowing is assumed to affect plant (Dorado and Lopez-Fando 2006, Gruber and Claupein 2009), carabid (Thorbek and Bilde 2004), and bird (Newton 2004) communities. Although mechanical weed control is more frequently used in organic farming, the effects of mechanical weed control on plant and arthropod communities can be severe (van Elsen 2000, Hatcher and Melander 2003, Thorbek and Bilde 2004) and were therefore included into the AI index. Based on this information, we calculated an AI index for each farm. Three agricultural practices, namely, pesticide input (number of applications; insecticides, herbicides, fungicides, and other pesticides), fertilizer input ( $\mathrm{kg} \mathrm{N}$ per year per hectare applied, both organic and inorganic), and the number of tillage operations and mechanical weeding were normalized, averaged, and added following Herzog et al. (2006):

$$
\mathrm{AI}=\frac{\sum_{i=1}^{n}\left(y_{i}-y_{i \min }\right) /\left(y_{i \max }-y_{i \min }\right)}{n} \times 100
$$

where $\mathrm{AI}$ is the agricultural intensification index, $y_{i}$ is the observed value (number of pesticide applications, amount of fertilizer applied, or number of tillage operations), $y_{\min }$ is the minimum observed value in all regions, $y_{\max }$ is the maximum observed value in all regions, $n$ is the number of individual indicators, and $i$ is the identifier for the three indicators.

Based on this AI index, each farm within a local $50 \times$ $50 \mathrm{~km}$ region was assigned to one level of agricultural intensification (low, medium, and high). The continuous variable AI was split into three discrete levels to allow for the calculation of $\beta$ diversities within each level on the region scale. Within each region, farms were classified into three groups into 10 farms of each AI level to avoid spatial autocorrelation of the AI levels over the whole study area (i.e., all regions; for mean AI index values per level, see Table 1). Otherwise, farms of one AI level might have been clumped in one or a few regions, and hence, it would not have been possible to separate AI level and region effects. To test for the effects of overlapping AI index values between AI levels and regions, we additionally analyzed a reduced data set with data only from regions with similar AI index values for the low, medium, and high AI treatments, i.e., France, Ireland, and Sweden were excluded, which 
TABLE 1. Mean ( \pm SE) agricultural intensification (AI) index values for low, medium, and high AI levels in each region and for all regions combined.

\begin{tabular}{lrcr}
\hline \hline \multirow{2}{*}{\multicolumn{1}{c}{ Region }} & \multicolumn{3}{c}{ Mean AI index } \\
\cline { 2 - 4 } \multicolumn{1}{c}{ Low AI } & Medium AI & High AI \\
\hline Estonia & $5.53 \pm 0.51$ & $15.03 \pm 1.46$ & $24.43 \pm 1.12$ \\
France & $13.54 \pm 0.73$ & $16.21 \pm 0.18$ & $18.04 \pm 0.33$ \\
Germany Göttingen & $3.07 \pm 0.73$ & $16.63 \pm 2.56$ & $32.11 \pm 2.28$ \\
Germany Jena & $13.63 \pm 1.72$ & $27.81 \pm 0.78$ & $36.42 \pm 1.96$ \\
Ireland & $29.74 \pm 1.21$ & $31.76 \pm 1.10$ & $33.06 \pm 0.97$ \\
Netherlands & $13.47 \pm 1.65$ & $22.39 \pm 0.70$ & $35.30 \pm 3.04$ \\
Poland & $14.98 \pm 1.22$ & $21.15 \pm 0.71$ & $30.71 \pm 1.95$ \\
Spain & $14.70 \pm 1.07$ & $20.87 \pm 0.56$ & $27.11 \pm 1.27$ \\
Sweden & $5.18 \pm 0.80$ & $13.20 \pm 0.49$ & $19.95 \pm 1.57$ \\
Overall mean & $12.65 \pm 0.84$ & $20.56 \pm 0.65$ & $28.57 \pm 0.70$ \\
\hline
\end{tabular}

yielded comparable results (Appendix B). Hence, we used the whole data set in the present analysis.

\section{Landscape structure}

Landscape structure in a $1000 \mathrm{~m}$ radius around the sampling points was measured on the basis of official topographical maps $(1: 5000$; DGK 5, Deutsche Grundkarte, Landesvermessung und Geobasisinformation Lower Saxony, Hannover, Germany) using ArcGIS 9.2 (ESRI 2006). The percentage of agricultural fields, which is closely related to habitat type diversity and mean field size, was used as an indicator for landscape complexity (Thies and Tscharntke 1999, Thies et al. 2003, Roschewitz et al. 2005). Because percentage arable land was highly correlated with mean field size, only the percentage of arable land was used in the analysis. Landscape structure on higher spatial scales was calculated using the mean values from the corresponding lower spatial scale. For birds, only the mean field size and the percentage of arable land within the $500 \times 500 \mathrm{~m}$ quadrats were measured.

\section{Additive partitioning of species diversity}

The total observed non-rarefied species richness, $\gamma_{\text {obs }}$, for each AI level and community can be partitioned as

$$
\gamma_{\text {obs }}=\alpha+\beta_{\text {field }}+\beta_{\text {farm }}+\beta_{\text {region }}
$$

where $\alpha$ is the mean $\alpha$ diversity per field, $\beta_{\text {field }}$ is the between-field $\beta$ diversity, $\beta_{\text {farm }}$ is the mean between-farm $\beta$ diversity, and $\beta_{\text {region }}$ is the mean between-region $\beta$ diversity. Because birds were sampled on the farm level, bird data could only be partitioned into $\gamma_{\mathrm{obs}}=\alpha_{\mathrm{farm}}+$ $\beta_{\text {farm }}+\beta_{\text {region }}$.

These values can be obtained as follows:

$$
\begin{aligned}
\alpha & =\frac{1}{n} \sum_{i j k} \alpha_{i j k} \\
\beta_{\text {field }} & =\frac{1}{n} \sum_{i j k}\left(\gamma_{j k}-\alpha_{i j k}\right)
\end{aligned}
$$

$$
\beta_{\mathrm{farm}}=\frac{1}{N} \sum_{j k}\left(\gamma_{k}-\gamma_{j k}\right)
$$

$$
\beta_{\text {region }}=\frac{1}{M} \sum_{k}\left(\gamma_{\mathrm{obs}}-\gamma_{k}\right)
$$

where $n$ is the total number of fields (450), $N$ is the total number of farms within regions (90), $M$ is the total number of regions (9), $i$ is the identifier for field within farm, $j$ is the identifier for farm within region (90), and $k$ is the identifier for each region (9) for each AI level.

We also calculated the relative $\alpha$ and $\beta$ diversities, i.e., the percentage of contribution of $\alpha$ and $\beta$ diversities to $\gamma_{\text {obs. }}$.

\section{Statistical models}

Linear mixed-effects models were used to test the effects of agricultural intensification and landscape structure on the $\alpha, \beta$, and $\gamma$ diversity of carabids, plants, and birds species richness. AI was included as a factor with three levels (low, medium, and high) and the percentage of arable land was included as a continuous variable. Alpha and beta diversities were tested separately at field, farm, and regional scales, respectively, i.e., one model for the effect of AI and landscape structure on the $\alpha$ diversity on the field scale, one model for the effect of AI and landscape structure on the $\beta$ diversity on the field scale, one model for the effect of AI and landscape structure on the $\beta$ diversity on the farm scale, and so on. In total, we analyzed 450 observations per AI level at the field scale, 90 calculations of $\beta$ diversities per AI level at the farm scale, and 9 calculations of $\beta$ diversities per AI level at the regional scale. There were 1350 total replications $(n)$ for the models at the field level, 270 at the farm level, and 27 on the region level. Because these observations were not independent from each other, fields were nested within farms, and farms were nested within regions and included as random factors into the models. We analyzed the effects of AI on the relative contribution using linear mixed-effect models with the same nesting structure. Model assumptions were checked 
TABLE 2. Effect of AI level (low, medium, and high) on the $\alpha$ and $\beta$ diversities of the three taxa.

\begin{tabular}{lrcccr}
\hline \hline \multirow{2}{*}{$\begin{array}{c}\text { Taxon } \\
\text { and scale }\end{array}$} & & & \multicolumn{3}{c}{ Species richness estimates } \\
\cline { 4 - 6 } & $\mathrm{df}$ & $F$ & Low AI & Medium AI & High AI \\
\hline Plants & & & & & \\
$\alpha$ & 253 & $15.23^{* * *}$ & $11.24^{\mathrm{a}}$ & $8.68^{\mathrm{b}}$ & $7.24^{\mathrm{b}}$ \\
$\beta_{\text {field }}$ & 253 & $5.08^{* *}$ & $9.80^{\mathrm{b}}$ & $8.89^{\mathrm{ab}}$ & $7.17^{\mathrm{a}}$ \\
$\beta_{\text {farm }}$ & 253 & $13.27^{* * *}$ & $44.04^{\mathrm{a}}$ & $44.39^{\mathrm{a}}$ & $38.30^{\mathrm{b}}$ \\
$\beta_{\text {region }}$ & 16 & $23.63^{* * *}$ & $232.92^{\mathrm{a}}$ & $220.84^{\mathrm{a}}$ & $195.74^{\mathrm{b}}$ \\
Carabids & & & & & \\
$\alpha$ & 253 & 2.51 n.s. & 6.39 & 6.30 & 5.80 \\
$\beta_{\text {field }}$ & 253 & 0.81 n.s. & 7.43 & 7.91 & 7.74 \\
$\beta_{\text {farm }}$ & 211 & $5.64^{* *}$ & $27.63^{\mathrm{a}}$ & $19.09^{\mathrm{b}}$ & $26.10^{\mathrm{a}}$ \\
$\beta_{\text {region }}$ & 16 & $5.85^{* *}$ & $133.8^{\mathrm{a}}$ & $127.4^{\mathrm{b}}$ & $126.2^{\mathrm{b}}$ \\
Birds & & & & 11.27 & \\
$\alpha$ & 213 & 2.73 n.s. & 12.61 & $12.99^{\mathrm{ab}}$ & 12.38 \\
$\beta_{\text {farm }}$ & 213 & $5.38^{* *}$ & $14.94^{\mathrm{a}}$ & $66^{\mathrm{b}}$ & $59.63^{\mathrm{b}}$ \\
$\beta_{\text {region }}$ & 14 & $7.98^{* *}$ & $72.88^{\mathrm{a}}$ & & \\
\hline
\end{tabular}

Notes: $F$ and $P$ values and denominator degrees of freedom $(\mathrm{df})$ of conditional $F$ tests performed on linear mixed-effect models are given. Numerator degrees of freedom are always 2 . Significant differences between species richness estimates are marked by different superscript letters. See Methods for a description of the scales.

$* * P<0.01 ; * * * P<0.001 ;$ "n.s." indicates not significant.

using diagnosis plots and dependent variables were either log- or square-root-transformed and/or variance functions (varIdent) were used (Pinheiro and Bates 2000) to account for non-normal distribution and heteroscedasticity when necessary. The significance of AI in each model was determined by using conditional $F$ tests, where the null model was tested against a model with AI.

We applied paired $t$ tests on the whole data set of each taxon, i.e., across all AI levels, to test for taxon-specific differences in the percentage contribution to total species richness. On each scale, one $t$ test per taxon combination was done. Post hoc Tukey hsd tests were applied to separate means. All analyses were performed using R 2.8.1 (R Development Core Team 2009) and the packages nlme (Pinheiro et al. 2009) and multcomp (Hothorn et al. 2008).

\section{Results}

Effects of AI level on $\alpha, \beta$, and $\gamma$ diversity of plants, carabids, and birds

In total, we recorded 423 vascular plant species, 219 carabid beetle species, and 121 bird species.

Comparisons of the $\alpha$ and $\beta$ diversities between low, medium, and high agricultural intensification (AI) revealed that the effects of $\mathrm{AI}$ on species richness were taxon specific. High AI significantly decreased $\alpha$ and $\beta$ diversities of plants at all scales, while $\beta$ diversities of carabids were only decreased at the region scale, and bird $\beta$ diversities were decreased at the farm and region scale (Table 2 and Fig. 2). The percentage of arable land was negatively related to plant and bird $\alpha$ diversity and bird $\beta_{\text {farm }}$ diversity. Carabid $\beta_{\text {farm }}$ diversity showed a significant interaction between AI and percentage of arable land. Only at the medium AI level was the
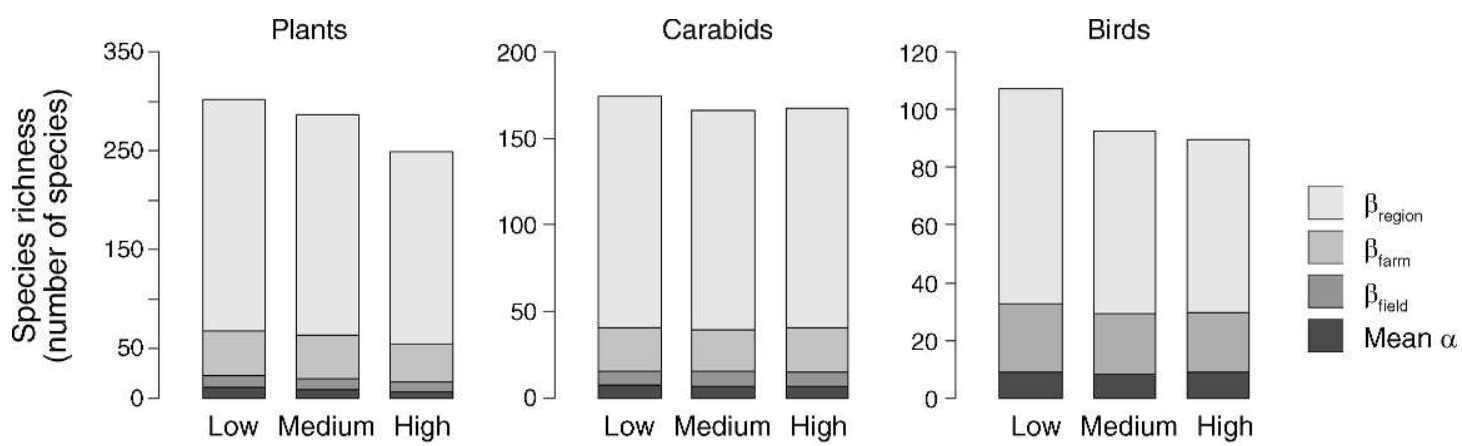

Agricultural intensification

FIG. 2. Effect of agricultural intensification (AI) level (low, medium, and high) on species richness of plants, carabids, and birds, separating $\alpha$ diversity on the field and $\beta$ diversity between fields, farms, and regions. 
TABLE 3. Correlation between percentage of arable land in a $1000 \mathrm{~m}$ radius around the plots and $\alpha$ and $\beta$ diversities of the three taxa and their interactions.

\begin{tabular}{|c|c|c|c|c|c|}
\hline Taxon & Scale & Variable & df & $F$ & Description \\
\hline Plants & $\alpha$ & percentage of arable land & 1035 & $6.73^{* *}$ & negative \\
\hline Carabids & $\beta_{\text {farm }}$ & interaction percentage arable land & 211 & $3.35^{*}$ & medium: positive \\
\hline Birds & $\alpha$ & percentage arable land & 213 & $9.95 * *$ & negative \\
\hline Birds & $\beta_{\text {farm }}$ & percentage arable land & 213 & $13.93 * * *$ & positive \\
\hline
\end{tabular}

Notes: $F$ and $P$ values and denominator degrees of freedom (df) of conditional $F$ tests performed on linear mixed-effect models are given. Numerator degrees of freedom are always 1 . The description column gives details about the direction of the main effects and interactions. "Interaction" is the interaction between percentage of arable land and AI. Only significant results are shown.

$* P<0.05 ; * * P<0.01 ; * * * P<0.001$.

percentage of arable land positively correlated to $\beta_{\text {farm }}$ diversity, while the low and high AI levels showed no relationship (Table 3 ).

\section{Effects of AI level on relative contribution of $\alpha, \beta$, and $\gamma$ diversity to total species richness of plants, carabids, and birds}

Shifts in relative contribution of $\alpha$ and $\beta$ diversity to the total species richness within AI levels, estimated as percentage of total diversity, were taxon specific (Table 4). Plants had a significantly larger relative contribution of $\alpha$ diversity at the low AI level, while carabids had higher relative $\beta_{\text {field }}$ diversity at the high and medium, compared to the low, AI level. Birds showed higher relative $\alpha$ diversity at medium and low, compared to high, AI level. The percentage of arable land was negatively related to relative plant and bird $\alpha$ diversity (Table 5).

\section{Effects of taxon on relative contribution of $\alpha$ and $\beta$ diversity to total species richness}

A comparison of the mean relative contribution to total diversity regardless of AI level on each scale between plants, carabids, and birds (Table 6) showed that the taxa in this study had significantly different relative contributions to total diversity, probably depending on their mobility. On the field scale, carabids showed higher $\alpha$ and $\beta$ diversity than plants. On the landscape scale, no differences between plants and carabids were apparent, while birds had higher $\beta$ diversity than carabids and plants. On the region scale, plants and carabids also did not differ, but both were higher than bird diversity. The importance of $\alpha$ and $\beta$ diversities for the species richness within each region is shown in Appendix C.

\section{Discussion}

The analysis of diversity patterns of plants, carabid beetles, and birds in agricultural landscapes across Europe showed that $\beta$ diversity between farms and regions contributed most to total diversity. Agricultural intensification (AI) was negatively correlated with species richness of plants and birds, whereas carabid beetles showed no such relationships, thus supporting our hypotheses that AI has a negative effect on species richness depending on taxon. AI, although based on local

TABLE 4. Effect of AI level on the relative contribution (percentage of total) of $\alpha$ and $\beta$ diversities to the $\gamma$ diversity of each taxa within each AI level.

\begin{tabular}{lrrrrr}
\hline \hline \multirow{2}{*}{$\begin{array}{c}\text { Taxon } \\
\text { and scale }\end{array}$} & & & \multicolumn{2}{c}{ Percentage of total species richness estimates } \\
\cline { 5 - 6 } & df & $F$ & Low AI & Medium AI & High AI \\
\hline Plants & & & & & \\
$\alpha$ & 253 & $6.99^{* *}$ & $3.82^{\mathrm{a}}$ & $3.09^{\mathrm{b}}$ & $2.92^{\mathrm{b}}$ \\
$\beta_{\text {field }}$ & 253 & 0.82 n.s. & 3.45 & 3.29 & 3.08 \\
$\beta_{\text {farm }}$ & 214 & 0.02 n.s. & 12.73 & 12.77 & 12.78 \\
$\beta_{\text {region }}$ & 16 & 0.15 n.s. & 77.63 & 77.66 & 78.58 \\
Carabids & & & & & 3.45 \\
$\alpha$ & 253 & 1.64 n.s. & 3.69 & 4.79 & 4.65 \\
$\beta_{\text {field }}$ & 253 & 2.39 n.s. & 4.29 & $12.18^{\mathrm{b}}$ & $16.46^{\mathrm{a}}$ \\
$\beta_{\text {farm }}$ & 211 & $6.37^{* *}$ & $16.11^{\mathrm{a}}$ & 76.31 & \\
$\beta_{\text {region }}$ & 16 & 0.48 n.s. & 76.95 & & 13.58 \\
Birds & & & & 12.64 & $23.79^{\mathrm{b}}$ \\
$\alpha$ & 213 & 0.56 n.s. & 12.78 & $21.77^{\mathrm{a}}$ & 68.53 \\
$\beta_{\text {farm }}$ & 219 & $8.57^{* * *}$ & $21.17^{\mathrm{a}}$ & 68.82 & \\
$\beta_{\text {region }}$ & 14 & 0.00 n.s. & 68.75 & & \\
\hline
\end{tabular}

Notes: $F$ and $P$ values and denominator degrees of freedom (df) of conditional $F$ tests performed on linear mixed-effect models are given. Numerator degrees of freedom are always 2. Significant differences between the percentage of total species richness estimates are marked by different superscript letters.

** $P<0.01 ; * * * P<0.001$; "n.s." indicates not significant. 
TABLE 5. Correlation between percentage of arable land in a $1000 \mathrm{~m}$ radius around the plots and relative contribution (percentage of total) of $\alpha$ and $\beta$ diversities to the $\gamma$ diversity of each taxa within each AI level.

\begin{tabular}{lccrrc}
\hline \hline Taxon & Scale & Variable & df & $F$ & Description \\
\hline Plants & $\alpha$ & percentage of arable land & 1035 & $6.98^{* * *}$ & negative \\
Birds & $\alpha$ & percentage of arable land & 213 & $12.74^{* * *}$ & negative \\
\hline
\end{tabular}

Notes: $F$ and $P$ values and denominator degrees of freedom (df) of conditional $F$ tests performed on linear mixed-effect models are given. Numerator degrees of freedom are always 1 . The description column gives details about the direction of the main effects and interactions. "Interaction" is the interaction between the percentage of arable land and AI. Only significant results are shown.

$$
\text { ** } P<0.01 ; * * * P<0.001 \text {. }
$$

practices, was closely related to the $\beta$ diversity on larger scales up to the region scale, with the detrimental effects of AI still recognizable on higher scales. Hence, AI at local scales appeared to be an indicator of farm- and region-wide loss of diversity.

\section{Effects of AI on species richness across different taxa}

The great role of $\beta$ diversity contribution to overall biodiversity at the regional scale has been shown by several studies (Wagner et al. 2000, Roschewitz et al. 2005, Gabriel et al. 2006, Clough et al. 2007, Hendrickx et al. 2007) and stress the huge importance of environmental heterogeneity at larger scales.

In this study, plant species richness was significantly reduced under intensive management. This detrimental effect of high input farming was consistent over all scales, indicating species poorer plant communities under high AI. This is supported by the literature, showing positive effects of low-input farming, i.e., reduced pesticide and/or reduced fertilizer input, on plant species richness (e.g., Stoate et al. 2001, Bengtsson et al. 2005, Gabriel et al. 2006, Liira et al. 2008, Kleijn et al. 2009). In addition, we could confirm that plant species richness was reduced with increasing landscape simplification (as shown by the percentage of arable land; Tscharntke et al. 2005, Gabriel et al. 2006).

In contrast, AI had no effect on the species richness of carabids on the field and farm scale, although detrimental effects of soil management, for example, plowing, mechanical weed control, or grass cutting on arthropods are well known (Holland and Reynolds 2003, Thorbek and Bilde 2004). However, pesticide use in crop edges usually does not seem to affect carabid species richness negatively (Frampton and Dorne 2007), although pesticides may change carabid abundance and community composition directly and indirectly (Shah et al. 2003, Navntoft et al. 2006, Geiger et al. 2010). Our results agree with studies showing no effect of AI on the species richness of carabids in cereal fields at the regional and local scale (Clough et al. 2007, Batáry et al. 2008, but see Geiger et al. 2010). Only at the region scale did a positive effect of lower AI on $\beta$-diversity exist, indicating more dissimilar and diverse carabid communities between regions under low AI management (also see Schweiger et al. 2005, Hendrickx et al. 2009). However, we found a significant interaction between AI and percentage of arable land. Here, an increasing amount of arable land slightly increased dissimilarity of communities only on the medium AI level.

Bird species richness was enhanced under low AI. This could be due to improved food and nesting resource availability in low-intensity management fields (Stephens et al. 2003) and more diverse and complex field margins (e.g., hedgerows, woodland), stressing the importance of physical heterogeneity on the field as an important determinant of bird diversity (Wilson et al. 2005). Granivorous birds especially could be affected through the loss of food and nesting resources through weed control at higher AI (Newton 2004). We also found a negative correlation between the percentage of arable land and bird $\alpha$ diversity and $\beta_{\text {farm }}$ diversity, highlighting the importance of seminatural habitats for the bird community in arable landscapes independently from local AI on the field.

\section{AI effects across different spatial scales}

If local AI determines the local diversity of a specific set of species out of the species pool, AI would enhance

TABLE 6. Results of paired $t$ tests comparing the relative contribution of each scale to taxonspecific total species richness between the different taxa (plants, P; carabids, C; birds, B).

\begin{tabular}{llrrc}
\hline \hline Scale & Compared taxa & $t$ & df & Direction of effect \\
\hline$\alpha$ & plants-carabids & $-14.07^{* * *}$ & 1315 & $\mathrm{P}<\mathrm{C}$ \\
$\beta_{\text {field }}$ & plants-carabids & $-10.95^{* * *}$ & 1315 & $\mathrm{P}<\mathrm{C}$ \\
$\beta_{\text {farm }}$ & plants-carabids & 1.24 n.s. & 263 & $\mathrm{P}=\mathrm{C}$ \\
$\beta_{\text {farm }}$ & plants-birds & $-12.13^{* * *}$ & 228 & $\mathrm{~B}>\mathrm{P}$ \\
$\beta_{\text {farm }}$ & carabids-birds & $-11.22^{* * *}$ & 228 & $\mathrm{~B}>\mathrm{C}$ \\
$\beta_{\text {region }}$ & plants-carabids & 0.97 n.s. & 26 & $\mathrm{P}=\mathrm{C}$ \\
$\beta_{\text {region }}$ & plants-birds & $3.68^{* * *}$ & 23 & $\mathrm{~B}>\mathrm{P}$ \\
$\beta_{\text {region }}$ & carabids-birds & $3.10^{* * *}$ & 23 & $\mathrm{~B}<\mathrm{C}$ \\
\hline
\end{tabular}

*** $P<0.001 ;$ n.s. is not significant. 
the relative importance (i.e., percentage contribution of $\alpha$ diversity to $\gamma$ diversity) of $\alpha$ diversity, homogenizing community composition between sites due to increasingly similar environmental conditions (Tylianakis et al. 2005, Quintero et al. 2010). In our study, AI on the field was not related to large-scale land-use changes, as AI was not significantly correlated with the percentage of arable land (with the exception of carabid $\beta_{\text {farm }}$ diversity). Hence, local effects of AI could be disentangled from effects such as landscape homogenization. In contrast to the expected enhancement of the relative importance of $\alpha$ diversity, local AI decreased the relative $\alpha$ diversity of plants and increased the relative importance of $\beta_{\text {farm }}$ diversity of birds and carabids under high AI, indicating an increased species turnover. Hence, AI does not necessarily homogenize local communities presumably due to heterogeneity of farming practices in intensified agriculture.

In addition, we could show that the percentage of arable land independent from AI was negatively correlated with the relative $\alpha$ diversity of plants and birds. Therefore, increasing landscape simplification was connected to more and more dissimilar communities, although this relationship was relatively weak as shown by the nonsignificant results concerning $\beta$ diversities.

\section{Differences in spatial diversity patterns between plants, carabids, and birds}

Species group identity had a significant effect on the spatial diversity patterns, indicated by a different partitioning of diversity components (relative $\alpha, \beta_{\text {field }}$, $\beta_{\text {farm, }}$, and $\left.\beta_{\text {region }}\right)$. One could expect birds to have, on average, a higher mobility and possibly higher dispersal ability than carabids. Both taxa are surely more mobile than plants as sessile organisms. The more mobile taxa had, on average, less similar communities on the field and farm level than plants (Table 4), i.e., $\alpha_{\text {field }}$ and $\beta_{\text {field }}$ represented a greater proportion of $\gamma$ diversity for carabids, and $\alpha_{\text {farm, }}, \beta_{\text {farm, }}$ and $\beta_{\text {region }}$ represented a greater proportion of $\gamma$ diversity for birds in comparison to plants. One possible explanation are spillover effects from the adjacent non-crop habitats for more mobile taxa, which could lead to variance in the local species composition (Hendrickx et al. 2009), as supported by several studies stressing the importance of the surrounding landscape and field margins for the within field carabid community (Denys and Tscharntke 2002, Purtauf et al. 2005, Schweiger et al. 2005, Hendrickx et al. 2007, Batáry et al. 2008, Smith et al. 2008). Likewise, the importance of species-rich field margins and seminatural habitats for within-field species richness has already been shown for many other arthropod taxa depending on body size (ability for mobility; Schmidt and Tscharntke 2005, Schmidt et al. 2005, Schweiger et al. 2005, Holland et al. 2009) and farmland birds (Vickery et al. 2002, Billeter et al. 2008). For plants, however, the picture could be a little different. The within-field environmental conditions between farms are likely more similar than the environmental conditions in the surrounding landscapes around these fields. For plants, as less mobile taxa, this could act as a homogenizing force, filtering out species with specific traits and life histories (Liira et al. 2008). Plant species richness might be more strongly affected by local management than by landscape and field margin composition and structure (Gabriel et al. 2006, Aavik et al. 2008, Marshall 2009), although landscape has a relevant effect, especially at high landscape complexity (Roschewitz et al. 2005, Gabriel et al. 2006, Aavik and Liira 2010). This is in line with results of a meta-analysis from Attwood et al. (2008), which show that the loss of species richness of arthropods from native to agricultural systems is greater than that through AI within agricultural systems.

Birds had a higher relative $\beta$ diversity on the farm scale than plants and carabids, indicating relatively more dissimilar communities. Because bird species richness was measured in a $500 \times 500 \mathrm{~m}$ square, sometimes not only the focal field, but parts of the surrounding landscape were sampled, too. However, AI and percentage of arable land in the $500 \times 500 \mathrm{~m}$ square were not correlated in our study, hence, disentangling AI and landscape effects.

At the region scale, birds had a significantly lower relative $\beta$ diversity than plants and carabids, indicating a possible homogenizing effect of dispersal ability on species richness (Mouquet and Loreau 2003, Cadotte 2006). With increasing dispersal ability, species turnover between plots should decrease, caused by community homogenization between plots through dispersal over long distances.

\section{Conclusions and management implications}

Our study shows that $\beta$ diversity accounted for the major part of the total diversity, an effect consistent over all nine European regions included in this study. Landscape simplification was negatively correlated to the $\alpha$ diversity of plants and birds and $\beta_{\text {farm }}$ diversity of birds. AI was correlated to decreased species richness of plants and birds on all scales, i.e., local AI did not only negatively affect the local diversity on the field, but was also related to reduced species turnover between fields, farms, and regions. In contrast, carabid beetle species richness was not correlated to AI, possibly due to a relatively high tolerance to pesticides and a high impact of immigration from surrounding field margins and seminatural habitats.

AI did not cause the hypothesized higher relative contribution of $\alpha$ than $\beta$ diversity to total diversity, which would have indicated a homogenizing effect of AI. Relative contributions of $\beta$ diversity to total diversity across all scales were taxon specific, highlighting the need to consider traits such as species mobility when planning agri-environmental schemes. This means, from an applied point of view, agri-environmental schemes need to (1) expand the view from the local field and farm to the landscape and region level to appropriately predict the effectiveness of agri-environ- 
mental schemes, shown by the huge contribution of $\beta$ diversity of large scales; and to (2) consider taxon specific responses to conservation efforts.

\section{ACKNOWLEDGMENTS}

We thank the people who did the bird surveys and Christoph Scherber for statistical advice. We also thank the European Science Foundation and the connected eight national science foundations for funding the presented study through the Eurodiversity AGRIPOPES (agricultural policy-induced landscape changes: effects on biodiversity and ecosystem services) program and the biology program BIOPLEX of the German Federal Ministry of Education and Science (BMBF).

\section{Literature Cited}

Aavik, T., I. Augenstein, D. Bailey, F. Herzog, M. Zobel, and J. Liira. 2008. What is the role of local landscape structure in the vegetation composition of field boundaries? Applied Vegetation Science 11:375-386.

Aavik, T., and J. Liira. 2010. Quantifying the effect of organic farming, field boundary type and landscape structure on the vegetation of field boundaries. Agriculture, Ecosystems and Environment 135:178-186.

Attwood, S. J., M. Maron, A. P. N. House, and C. Zammit. 2008. Do arthropod assemblages display globally consistent responses to intensified agricultural land use and management? Global Ecology and Biogeography 17:585-599.

Batáry, P., A. Kovacs, and A. Baldi. 2008. Management effects on carabid beetles and spiders in Central Hungarian grasslands and cereal fields. Community Ecology 9:247-254.

Bengtsson, J., J. Ahnstrom, and A. C. Weibull. 2005. The effects of organic agriculture on biodiversity and abundance: a meta-analysis. Journal of Applied Ecology 42:261-269.

Benton, T. G., J. A. Vickery, and J. D. Wilson. 2003. Farmland biodiversity: is habitat heterogeneity the key? Trends in Ecology and Evolution 18:182-188.

Bibby, J. C., D. N. Burgress, and A. D. Hill. 1992. Bird census techniques. Academic Press, London, UK.

Billeter, R., et al. 2008. Indicators for biodiversity in agricultural landscapes: a pan-European study. Journal of Applied Ecology 45:141-150.

Cadotte, M. W. 2006. Dispersal and species diversity: a metaanalysis. American Naturalist 167:913-924.

Chandy, S., D. J. Gibson, and P. A. Robertson. 2006. Additive partitioning of diversity across hierarchical spatial scales in a forested landscape. Journal of Applied Ecology 43:792-801.

Clough, Y., A. Holzschuh, D. Gabriel, T. Purtauf, D. Kleijn, A. Kruess, I. Steffan-Dewenter, and T. Tscharntke. 2007. Alpha and beta diversity of arthropods and plants in organically and conventionally managed wheat fields. Journal of Applied Ecology 44:804-812.

Collins, S. L., S. M. Glenn, and J. M. Briggs. 2002. Effect of local and regional processes on plant species richness in tallgrass prairie. Oikos 99:571-579.

Crawley, M. J., and J. E. Harral. 2001. Scale dependence in plant biodiversity. Science 291:864-868.

Crist, T., J. Veech, J. Gering, and K. Summerville. 2003. Partitioning species diversity across landscapes and regions: a hierarchical analysis of alpha, beta, and gamma diversity. American Naturalist 162:734-743

Denys, C., and T. Tscharntke. 2002. Plant-insect communities and predator-prey ratios in field margin strips, adjacent crop fields, and fallows. Oecologia 130:315-324.

Diekötter, T., R. Billeter, and T. O. Crist. 2008. Effects of landscape connectivity on the spatial distribution of insect diversity in agricultural mosaic landscapes. Basic and Applied Ecology 9:298-307.

Dorado, J., and C. Lopez-Fando. 2006. The effect of tillage system and use of a paraplow on weed flora in a semiarid soil from central Spain. Weed Research 46:424-431.
ESRI. 2006. ArcGIS. Version 9.2. ESRI, Redlands, California, USA.

Foley, J. A., et al. 2005. Global consequences of land use. Science 309:570-574.

Frampton, G. K., and J. Dorne. 2007. The effects on terrestrial invertebrates of reducing pesticide inputs in arable crop edges: a meta-analysis. Journal of Applied Ecology 44:362-373.

Gabriel, D., I. Roschewitz, T. Tscharntke, and C. Thies. 2006. Beta diversity at different spatial scales: plant communities in organic and conventional agriculture. Ecological Applications 16:2011-2021.

Geiger, F., et al. 2010. Persistent negative effects of pesticides on biodiversity and biological control potential on European farmland. Basic and Applied Ecology 11:97-105.

Gering, J. C., T. O. Crist, and J. A. Veech. 2003. Additive partitioning of species diversity across multiple spatial scales: implications for regional conservation of biodiversity. Conservation Biology 17:488-499.

Gough, L., C. W. Osenberg, K. L. Gross, and S. L. Collins. 2000. Fertilization effects on species density and primary productivity in herbaceous plant communities. Oikos 89:428-439.

Gruber, S., and W. Claupein. 2009. Effect of tillage intensity on weed infestation in organic farming. Soil and Tillage Research 105:104-111.

Hatcher, P. E., and B. Melander. 2003. Combining physical, cultural and biological methods: prospects for integrated non-chemical weed management strategies. Weed Research 43:303-322.

Hendrickx, F., J. P. Maelfait, K. Desender, S. Aviron, D. Bailey, T. Diekötter, L. Lens, J. Liira, O. Schweiger, M. Speelmans, V. Vandomme, and R. Bugter. 2009. Pervasive effects of dispersal limitation on within- and amongcommunity species richness in agricultural landscapes. Global Ecology and Biogeography 18:607-616.

Hendrickx, F., et al. 2007. How landscape structure, land-use intensity and habitat diversity affect components of total arthropod diversity in agricultural landscapes. Journal of Applied Ecology 44:340-351.

Herzog, F., et al. 2006. Assessing the intensity of temperate European agriculture at the landscape scale. European Journal of Agronomy 24:165-181.

Holland, J. M., T. Birkett, and S. Southway. 2009. Contrasting the farm-scale spatio-temporal dynamics of boundary and field overwintering predatory beetles in arable crops. Biocontrol 54:19-33.

Holland, J. M., and C. J. M. Reynolds. 2003. The impact of soil cultivation on arthropod (Coleoptera and Araneae) emergence on arable land. Pedobiologia 47:181-191.

Hothorn, T., F. Bretz, and P. Westfall. 2008. Simultaneous inference in general parametric models. Biometrical Journal 50:346-363.

Kleijn, D., et al. 2009. On the relationship between farmland biodiversity and land-use intensity in Europe. Proceedings of the Royal Society of London B 276:903-909.

Lande, R. 1996. Statistics and partitioning of species diversity, and similarity among multiple communities. Oikos 76:5-13.

Legendre, P., D. Borcard, and P. R. Peres-Neto. 2005. Analyzing beta diversity: partitioning the spatial variation of community composition data. Ecological Monographs $75: 435-450$.

Liira, J., et al. 2008. Plant functional group composition and large-scale species richness in the agricultural landscapes of Europe. Journal of Vegetation Science 19:3-14.

Marshall, E. J. P. 2009. The impact of landscape structure and sown grass margin strips on weed assemblages in arable crops and their boundaries. Weed Research 49:107-115.

Matson, P. A., W. J. Parton, A. G. Power, and M. J. Swift. 1997. Agricultural intensification and ecosystem properties. Science 277:504-509. 
Mouquet, N., and M. Loreau. 2003. Community patterns in source-sink metacommunities. American Naturalist 162:544557.

Navntoft, S., P. Esbjerg, and W. Riedel. 2006. Effects of reduced pesticide dosages on carabids (Coleoptera: Carabidae) in winter wheat. Agricultural and Forest Entomolgy 8:57-62.

Newton, I. 2004. The recent declines of farmland bird populations in Britain: an appraisal of causal factors and conservation actions. Ibis 146:579-600.

Pinheiro, J. C., and D. M. Bates. 2000. Mixed-effects models in $\mathrm{S}$ and S-PLUS. First edition. Springer, New York, USA.

Pinheiro, J. C., D. M. Bates, S. DebRoy, and D. Sarkar, and the R Core Team. 2009. nlme: linear and nonlinear mixed effects models. R package version 3.1-93. R Foundation for Statistical Computing, Vienna, Austria.

Purtauf, T., I. Roschewitz, J. Dauber, C. Thies, T. Tscharntke, and V. Wolters. 2005. Landscape context of organic and conventional farms: influences on carabid beetle diversity. Agriculture Ecosystems and Environment 108:165-174.

Pysek, P., and J. Leps. 1991. Response of a weed community to nitrogen-fertilization: a multivariate-analysis. Journal of Vegetation Science 2:237-244.

Quintero, C., C. L. Morales, and M. A. Aizen. 2010. Effects of anthropogenic habitat disturbance on local pollinator diversity and species turnover across a precipitation gradient. Biodiversity and Conservation 19:257-274.

R Development Core Team. 2009. R: a language and environment for statistical computing. R Foundation for Statistical Computing, Vienna, Austria.

Reich, P. B. 2009. Elevated $\mathrm{CO}_{2}$ reduces losses of plant diversity caused by nitrogen deposition. Science 326:1399-1402.

Robinson, R. A., and W. J. Sutherland. 2002. Post-war changes in arable farming and biodiversity in Great Britain. Journal of Applied Ecology 39:157-176.

Roschewitz, I., D. Gabriel, T. Tscharntke, and C. Thies. 2005. The effects of landscape complexity on arable weed species diversity in organic and conventional farming. Journal of Applied Ecology 42:873-882.

Schmidt, M., Y. Clough, W. Schulz, A. Westphalen, and T. Tscharntke. 2006. Capture efficiency and preservation attributes of different fluids in pitfall traps. Journal of Arachnology 34:159-162.

Schmidt, M. H., I. Roschewitz, C. Thies, and T. Tscharntke. 2005. Differential effects of landscape and management on diversity and density of ground-dwelling farmland spiders. Journal of Applied Ecology 42:281-287.

Schmidt, M. H., and T. Tscharntke. 2005. The role of perennial habitats for Central European farmland spiders. Agriculture Ecosystems and Environment 105:235-242.

Schweiger, O., et al. 2005. Quantifying the impact of environmental factors on arthropod communities in agricultural landscapes across organizational levels and spatial scales. Journal of Applied Ecology 42:1129-1139.

Shah, P. A., D. R. Brooks, J. E. Ashby, J. N. Perry, and I. P. Woiwod. 2003. Diversity and abundance of the coleopteran fauna from organic and conventional management systems in southern England. Agricultural and Forest Entomolgy 5:5160.

Smith, J., S. G. Potts, B. A. Woodcock, and P. Eggleton. 2008. Can arable field margins be managed to enhance their biodiversity, conservation and functional value for soil macrofauna? Journal of Applied Ecology 45:269-278.

Stephens, P. A., R. P. Freckleton, A. R. Watkinson, and W. J. Sutherland. 2003. Predicting the response of farmland bird populations to changing food supplies. Journal of Applied Ecology 40:970-983.

Stoate, C., N. D. Boatman, R. J. Borralho, C. R. Carvalho, G. R. de Snoo, and P. Eden. 2001. Ecological impacts of arable intensification in Europe. Journal of Environmental Management 63:337-365.

Thies, C., I. Steffan-Dewenter, and T. Tscharntke. 2003. Effects of landscape context on herbivory and parasitism at different spatial scales. Oikos 101:18-25.

Thies, C., and T. Tscharntke. 1999. Landscape structure and biological control in agroecosystems. Science 285:893-895.

Thorbek, P., and T. Bilde. 2004. Reduced numbers of generalist arthropod predators after crop management. Journal of Applied Ecology 41:526-538.

Tscharntke, T., A. M. Klein, A. Kruess, I. Steffan-Dewenter, and C. Thies. 2005. Landscape perspectives on agricultural intensification and biodiversity - ecosystem service management. Ecology Letters 8:857-874.

Tylianakis, J. M., A. M. Klein, T. Lozada, and T. Tscharntke. 2006. Spatial scale of observation affects alpha, beta and gamma diversity of cavity-nesting bees and wasps across a tropical land-use gradient. Journal of Biogeography 33:12951304.

Tylianakis, J. M., A. M. Klein, and T. Tscharntke. 2005. Spatiotemporal variation in the diversity of hymenoptera across a tropical habitat gradient. Ecology 86:3296-3302.

van Elsen, T. 2000. Species diversity as a task for organic agriculture in Europe. Agriculture Ecosystems and Environment 77:101-109.

Veech, J., K. Summerville, T. Crist, and J. Gering. 2002. The additive partitioning of species diversity: recent revival of an old idea. Oikos 99:3-9.

Vickery, J., N. Carter, and R. J. Fuller. 2002. The potential value of managed cereal field margins as foraging habitats for farmland birds in the UK. Agriculture Ecosystems and Environment 89:41-52.

Wagner, H. H., O. Wildi, and K. C. Ewald. 2000. Additive partitioning of plant species diversity in an agricultural mosaic landscape. Landscape Ecology 15:219-227.

Weiher, E., and A. Howe. 2003. Scale-dependence of environmental effects on species richness in oak savannas. Journal of Vegetation Science 14:917-920.

Wilson, J. D., M. J. Whittingham, and R. B. Bradbury. 2005. The management of crop structure: a general approach to reversing the impacts of agricultural intensification on birds? Ibis 147:453-463.

\section{APPENDIX A}

Details on the breeding-bird sampling protocol (Ecological Archives A021-080-A1).

\section{APPENDIX B}

Results of the analysis with a reduced data set (Ecological Archives A021-080-A2).

\section{APPENDIX C}

The $\alpha$ and $\beta$ diversities within each region disregarding agricultural intensification (Ecological Archives A021-080-A3). 